\title{
Medios de comunicación para la apropiación del conocimiento en instituciones educativas
}

Reynier I. Ramírez ${ }^{*}$, José V. Villalobos ${ }^{2}$, Nelson D. Lay ${ }^{3}$ y Belina A. Herrera ${ }^{4}$

(1) Universidad de la Costa, Dpto. de Ciencias Empresariales, Atlántico, Colombia (correo-e: rramirez13@cuc.edu.co).

(2) Universidad Pedro de Valdivia, Santiago, Chile (correo-e: jvvillalobos@gmail.com).

(3) Universidad Andres Bello, Escuela de Psicología, Viña del Mar - Chile (correo-e: nelson.lay@unab.cl).

(4) Universidad de la Costa, Dpto. de Derecho y Ciencias Políticas, Atlántico, Colombia.

(correo-e: bherrera3@cuc.edu.co).

* Autor a quien debe ser dirigida la correspondencia.

Recibido Jun. 17, 2020; Aceptado Ago. 14, 2020; Versión final Sep. 15, 2020, Publicado Feb. 2021

\begin{abstract}
Resumen
El objetivo de esta investigación fue examinar la descripción de los medios de comunicación para la apropiación del conocimiento en instituciones educativas del departamento de Magdalena, Colombia. Para tal fin, se realizaron diez entrevistas semiestructuradas, analizadas mediante la teoría fundamentada. Los resultados obedecen a la utilización de medios en espacios de aprendizajes para generar competencias (utilización, interacción y participación) del ser humano en sesiones de aprendizajes significativos. Los resultados muestran que el proceso de comunicación depende del perfil y labor del docente, número de participantes, herramientas didácticas disponibles y estructura de la institución, ocasionando barreras y filtros que limitan el aprendizaje. La apropiación del conocimiento está influenciada por modelos interactivos, con perspectivas emergentes sobre el saber. Se concluye que los medios de comunicación educativos se ven impactados por la cultura tecnológica del docente-estudiante, originando brechas de conocimiento para su adecuado uso.
\end{abstract}

Palabras clave: medios de comunicación; medios educativos; apropiación del conocimiento

\section{Media for the appropriation of knowledge in educational institutions}

\begin{abstract}
The objective of this research study was to assess how media describes the appropriation of knowledge in educational institutions in the state of Magdalena (Colombia). Ten semi-structured interviews were conducted and analyzed using grounded theory. The results obtained respond to the use of means in learning spaces to generate competencies (use, interaction, and participation) of the human being in meaningful learning sessions. The results show that the communication process depends on the profile and work of the teacher, number of participants, available teaching tools, and structure of the institution. These create barriers and filters that limit learning. Interactive models, with emerging perspectives on knowledge, influence knowledge appropriation. It is concluded that educational media are affected by the teacher-student's technological culture, which can cause knowledge gaps for proper educational media use.
\end{abstract}

Keywords: communication media; educational media; knowledge appropriation 


\section{INTRODUCCIÓN}

Con el pasar del tiempo, el ser humano es informado por medios de comunicación, que dan a conocer acontecimientos y aspectos relevantes de la esfera social. Estos han creado formas de acción y tipos de interacción social derivados de enfoques emergentes, posibilitando la apropiación del conocimiento, permitiendo transmitir información o contenidos simbólicos, para potenciales receptores tanto presencial como virtual, intervenidos por la tecnología de la información y comunicación (TIC), en un mundo global. Como dice Félix (2014), las nuevas TIC se pueden entender como una respuesta a las exigencias de la globalización, facilitando procesos interactivos, en busca de la productividad y nuevos aprendizajes. La interacción presencial se da en un contexto de co-presencia y espacio-temporal común; la interacción virtual implica el uso de medios técnicos para transmitir contenidos simbólicos a individuos, indiferentemente del referente espacio-tiempo. Con el desarrollo industrial de la impresión en los siglos XV y XVI, medios electrónicos en los siglos XIX y XX, y recientemente los digitales o nuevas medias, han asumido importantes fuentes de información interactivas, sobre todo para la apropiación del conocimiento, (Rodríguez-Hoyos y Fueyo, 2018). Hablar de los medios de comunicación, se relaciona con la historización, contrastes que determinan propiedades específicas de la modernidad de estos, como fenómeno del siglo XX (Martín, 2019), donde organizaciones operaban con herramientas indisolubles de las estructuras políticas, que por tanto ya estaban en algunas dimensiones de su praxis interactiva.

Por lo tanto, el concepto comunicación describe la interacción de procesos de una influencia interna ejercida, en determinados campos de prácticas (personales, culturales, políticas, educativas, públicas y privadas) (Määttä et al., 2017; Félix, 2014), y los medios, fuerzas singulares externas, aliens intrusos en las diversas esferas de la vida (Lin y Wei, 2019). Ante estos enfoques teóricos, es necesario entender los medios de comunicación de acuerdo al: (a) proceso y (b) el medio, dado por la realidad cronológica de posturas epistemológicas del término, posibilitando la apropiación del conocimiento en un contexto educativo. Ante los aportes referidos, los medios de comunicación para la apropiación del conocimiento se asocian a los medios (vías, temas, contenidos y contenedores) y procesos de mediar entre dos o más elementos, proximidad a una cosa, en términos de tiempo, lugar o grado, para consolidar aprendizajes significativos. Siendo las maneras o metodologías usadas por los educadores y estudiantes para viabilizar el conocimiento, presencial, corporal o verbal, digitalizado, en lugares de encuentro de organizaciones sociales, culturales y educativas, o en otros escenarios del aprendizaje, ya sean privados, públicos o mixtos.

Mediar el conocimiento, implica el uso de las TIC, como el recurso convencional o herramienta educativa, ambos se convierten en un medio de comunicación, su diferencia está en lo sincrónico y asincrónico. En algunos actos educativos se puede prescindir de estos insumos, sin restarle mérito al termino mediado, la voz y el cuerpo pueden usarse como medios, espacio territorial - distancia espacial, gestualidad - oralidad, de los docentes para gestionar los procesos de aprendizajes. Por su parte, Eze et al., (2018), sugiere el acceso a dispositivos móviles, ordenadores de última generación con acceso a internet, televisores, video proyectores, infraestructura de telecomunicaciones al servicio de la educación, con lo cual bastaría la dotación y alfabetización tecnológica para garantizar el aprendizaje, reduciendo la brecha del acceso a la tecnología, pero no la brecha de alfabetización tecnológica y educación para los medios de comunicación, menos aún el uso educativo de los medios y las TIC. La era global, información e hiperconectividad, exige a las instituciones educativas una sólida capacidad productiva para la apropiación del conocimiento, adaptabilidad al cambio e inclusión de la tecnología, asegurando su desarrollo (Cabero y Llorente, 2010; Gómez-Rodríguez et al., 2017), por lo tanto, demanda una actitud de apertura, flexibilidad, receptividad a las necesidades, expectativas de sus grupos de interés, aprendizaje compartido y uso adecuado de los medios de comunicación.

Los medios de comunicación para la apropiación de conocimiento, se han convertido en herramientas educativas de difusión de productos simbólicos en las instituciones, utilizando las TIC en los procesos de enseñanza significativa, dinamizando estrategias pedagógicas e incentivando al estudiante, aperturando nuevos roles y modelos de aprendizajes, siendo los medios de comunicación los productores de cultura mediada, posibilitando la apropiación de hechos y construcción de significados para la comprensión y explicación del entorno. Schwartz (2015), en su estudio sobre los enfoques de conocimiento para la apropiación de nuevos medios en una escuela, sostiene que la tecnología es subutilizada en las escuelas, en lugar de reflejar creatividad e innovación, se debe hacer énfasis en una nueva alfabetización y multialfabetismo técnico, con medios para apropiar y desarrollar conocimiento. Ante estas circunstancias, hay nuevas oportunidades de aprendizaje, desaprendizaje, pero también de reaprendizaje del proceso educativo permanente. La telemática y su naturaleza social, se ha caracterizado por interconectar personas y máquinas de manera constante (Valcke et al., 2010; McQuail, 1997).

Los medios de comunicación, actualmente indican el desarrollo futuro de herramientas con aplicaciones móviles, gestión del cambio en el contexto social académico, minimizando sus resistencias naturales, influenciadas por factores humanos y no humanos, siendo este, el papel crítico y dinámico de diversos actores 
en la red socio técnica, para la apropiación del conocimiento. Para Martín (2019) y Eze et al., (2018), se hace necesario conocer la realidad de la presencia de los medios de comunicación en las instituciones educativas para concienciar al ciudadano del uso adecuado de los nuevos medios, impactando sobre sus estándares de vida, acercándose a la comunicación productiva sincrónica y asincrónica. En la apropiación de conocimiento de las instituciones educativas se evidencian nuevos modelos interactivos, perfiles y tendencias, originando novedosos constructos para aprender colaborativamente, con perspectivas innovadoras sobre la metodología para el saber y hacer, influenciado por la tecnología, y ésta varía, por la cultura de sus públicos (Bartau-Rojas et al., 2018; Chassiakos et al., 2016; Lee, 2016), la metodología en entornos de aprendizaje, optimiza la interacción social como favorecedora del cambio socio-cognitivo.

\section{FENÓMENO BAJO ESTUDIO}

En un estudio realizado por Area-Moreira et al., (2016), manifiestan que el perfil del profesorado que desarrolla un modelo didáctico intensivo de las TIC es un docente con años de experiencia profesional, ciudadano usuario habitual de las TIC, suficientemente formado y con competencias digitales. Al respecto se hace necesario entonces fortalecer el apoyo al aprendizaje estudiantil y creativo, experiencias de aprendizaje propias a los medios emergentes, competencias al modelo educativo y responsabilidad adaptado a la era digital (Prendes y Gutiérrez, 2013). Es necesario profundizar en la formación del estudiante en relación a la competencia digital, reduciendo la brecha digital, la sociedad del conocimiento ha generado cambios epistemológicos y estructurales en todos los ámbitos de la vida, haciendo énfasis en los medios y procesos para aprender (Pérez-Escoda et al., 2016). El uso adecuado de los medios de comunicación para apropiar conocimiento se hace vital, los social media son considerados recursos que ayudan al aumento de las capacidades de relacionamiento e información.

El efecto de los medios de comunicación utilizados de manera adecuada, ostenta la capacidad para facilitar educación y aprendizaje colaborativo. La desinformación o información mal comunicada, origina comportamientos negativos y resultados adversos para la apropiación del conocimiento, (Villalobos et al., 2019; Livingstone et al., 2018; Jiménez-Iglesias et al., 2015). Al respecto, los procesos de comunicación desarticulados y uso desfavorable de los medios, desvinculado a los factores de comunicación en el plan de desarrollo, desarmonizado con las políticas nacionales, infraestructuras con falencias tecnológicas para asumir los procesos y el sistema de información del sector educativo, se perfilan dentro de las causas que afectan la apropiación del conocimiento en las instituciones educativas. En consecuencia, las debilidades antes mencionadas y sus incidencias en el proceso de mediación para la apropiación del conocimiento, ocasionan filtros que dificultan la transmisión y comprensión de mensajes recibidos, afectando significativamente la retroalimentación entre los interlocutores, imposibilitando la generación de conocimiento. Ante las cuestiones planteadas, en relación con la línea temática se hace la siguiente reflexión: ¿Cuáles son los medios de comunicación que utilizan los docentes para que los estudiantes apropien conocimiento?, por tal motivo, se describen los medios de comunicación para la apropiación del conocimiento en instituciones educativas del departamento de Magdalena - Colombia. En la tabla 1 se muestra una descripción de las categorías a tomar en cuenta como elementos de análisis de los medios de comunicación para la apropiación del conocimiento, contemplados durante la realización de este trabajo de investigación.

Tabla 1: Descripción de categorías como elementos de análisis de los medios de comunicación para la apropiación del conocimiento

\begin{tabular}{|l|l|}
\hline Categorías & Elementos de análisis \\
\hline Medios de comunicación. & $\begin{array}{l}\text { Funciones de los medios de comunicación social (informar, educar, entretener y formar } \\
\text { opinión). Nuevos medios de comunicación social: digital (audiovisuales y radiofónicos). }\end{array}$ \\
\hline Proceso de comunicación. & $\begin{array}{l}\text { Interacción sincrónica / asincrónica, mediante el emisor, receptor, mensaje, canal, } \\
\text { código, contexto, retroalimentación (sincrónica / asincrónica) y barreras. }\end{array}$ \\
\hline Alfabetización tecnológica. & Desarrollo de competencias tecnológicas: instrumentales, interpersonales y sistémicas. \\
\hline
\end{tabular}

\section{METODOLOGÍA}

En esta parte se describe el método de la teoría fundamentada utilizado en la investigación, por tal motivo se facilita una serie de pasos para consolidar el objetivo planteado, haciendo mención al: proceso de recopilación de datos, preguntas de investigación, participantes, recolección y análisis de los datos. Para Strauss y Corbin (2008), la teoría fundamentada es una metodología generalizada para desarrollar teoría sobre un fenómeno de estudio en particular, basándose en datos recolectados y analizados sistemáticamente, evolucionando durante el proceso de investigación, a través de la interacción continua entre el análisis y recopilación de datos. Glaser y Strauss (2017) y Sandín (2003), manifiestan que esta identifica categorías teóricas derivadas de los datos, utilizando un método comparativo e interactivo del contenido de las sesiones de entrevistas con los conceptos teóricos nacientes del esfuerzo por identificar los temas fundamentales. 
El proceso de investigación se basó en la teoría fundamentada descrita por Glaser y Strauss (2017): (1) revisión de la literatura, (2) identificación del problema, (3) preguntas de investigación, (4) guía de entrevista, (5) recolección y análisis de datos, y la (6) generación de teoría. De acuerdo a las preguntas de investigación, Martínez (2009), menciona que muchos investigadores con enfoque en los estudios cualitativos ven una simple pregunta como un punto de partida para su investigación, una vez que la pregunta se encuentra situada satisfactoriamente en su lugar, el estudio puede comenzar. Luego de la detección del problema de estudio, se identificaron tres preguntas de investigación: (1) ¿Cuál es la percepción de los docentes sobre los nuevos medios de comunicación social para la apropiación del conocimiento en instituciones educativas del departamento de Magdalena?; (2) ¿Cómo es el proceso de comunicación para la apropiación del conocimiento en instituciones educativas del departamento de Magdalena?; (3) ¿Cuentan los docentes con competencias tecnológicas para el adecuado uso de los medios de comunicación educativos para la apropiación del conocimiento?, buscando explorar elementos que toman en cuenta los docentes al momento de estimar los medios de comunicación para la apropiación del conocimiento en instituciones educativas del departamento de Magdalena - Colombia.

Al considerar los participantes, se empleó el método del muestreo selectivo propio de la investigación cualitativa. Martínez-Salgado (2012), menciona que en la indagación cualitativa, se eligen las unidades de estudio, para lograr un conocimiento profundo y detallado de casos en los que tiene lugar el fenómeno de interés, generalizable para otras situaciones en las que dicho fenómeno ocurre. Fueron 18 semanas las utilizadas para realizar el estudio, entre el período 2018-II (febrero-junio), caracterizado por ser abierto y exhaustivo, testimonios ajustados a las experiencias del interlocutor. Por ello, se optó por establecer un número limitado de diez sujetos, codificado de R1 al R10. Entre los docentes, el $40 \%$ hombres y el $60 \%$ mujeres, los criterios de inclusión: profesionales en educación, personal fijo, ambos sexos, 32 a 46 años de edad, indiferentemente de su estado civil, entre 8 a 16 años de experiencia laboral. De acuerdo a los criterios de exclusión de las unidades de estudio, se justifica por el decreto 2700 del Ministerio Educación Nacional, donde expone en el Artículo $2^{\circ}$, los requisitos que un municipio debe acreditar para ser certificados: (a) plan de desarrollo municipal armónico con las políticas nacionales; (b) establecimientos educativos organizados para ofrecer el ciclo de educación básica completa; (c) planta de personal definida de acuerdo con los parámetros nacionales; (d) capacidad institucional, para asumir los procesos y el sistema de información del sector educativo. De los entrevistados se tuvieron que descartar dos por falta de consistencia en la información tratada (R5 y R6). Se identificó que las 8 entrevistas restantes compartían pertinente y coherente información, lo que hacía posible reducir el número de entrevistas analizadas. Finalmente se eligieron cuatro para llevar a cabo el análisis cualitativo, debido a la calidad de la información proporcionada, en la similitud de ideas y rango de edad de los participantes (32-38 años), los docentes seleccionados para el análisis fueron R1, R4, R7 y R9.

Para la recolección de datos, se diseñó una guía de entrevista semiestructurada, tomando como base las preguntas de investigación antes mencionadas. Strauss y Corbin (2008), manifiestan que esto permite proporcionar un conjunto claro de instrucciones para los entrevistadores, generando datos cualitativos fiables y comparables, oportunidad de identificar nuevas formas de ver y comprender el tema bajo estudio. Para Martínez-Salgado (2012), este tipo de entrevista alienta al sujeto entrevistado para que narre algún evento importante de su vida y del contexto social. La guía de entrevista llevada a cabo se desarrolló con: (a) las instrucciones, (b) datos sociodemográficos, (c) percepción de los docentes, respecto a los medios de comunicación para la apropiación del conocimiento en instituciones educativas del departamento de Magdalena - Colombia, (d) indagación de los medios y procesos, que utilizan los docentes para que los estudiantes apropien conocimiento, (e) el cierre de la entrevista, con espacio para agregar comentarios oportunos, contribuyendo a la homogeneidad de lo estudiado. Investigadores y participantes, al encontrarse en departamentos diferentes, las entrevistas fueron llevadas a cabo a distancia, mediante videoconferencia, utilizando como vehículo de comunicación Skype. La grabación de las entrevistas se realizó con el sistema de grabación Recorder para el dispositivo Ipad, con duración aproximada de 45 y $1 \mathrm{~h} 30$ minutos.

Una vez concluidas las entrevistas, se realizó el análisis de los datos, para esto se efectuó la transcripción con el software InqScribe, y procesadas con el programa Atlas.Ti, versión 8.1, procurando el análisis línea por línea, permitiendo profundizar en las historias compartidas por los docentes, en busca de información relevante que responda a las preguntas de investigación planteadas, para ir generando la teoría a partir de estos. El proceso de análisis, inicia con la codificación abierta, encargada de descomponer la información línea por línea, la codificación axial sugiere la creación de categorías, en la codificación selectiva se elige una categoría base y, por último, se genera una teoría, todo esto validado por el juicio de expertos pertenecientes al grupo de investigación Administración Social, de la Universidad de la Costa.

En la codificación abierta, se identifican las ideas representadas en cada línea de texto transcritas, dándole un nombre clave a la nueva idea, preferentemente que sean palabras claras y concisas (Martínez, 2009). 
Incluye conceptos de etiquetado, definiendo y desarrollando categorías basadas en propiedades y dimensiones, durante este proceso, los conceptos surgen de datos brutos y luego se agrupan en categorías conceptuales, a fin de construir un marco preliminar descriptivo y multidimensional para su análisis posterior, el proceso asegura la validez del trabajo debido a que se construye directamente a partir de los datos brutos. La codificación axial, según Strauss y Corbin (2008), relaciona categorías a sus subcategorías, denominado "axial" porque la codificación ocurre alrededor del eje de una categoría, enlazando las categorías de acuerdo a sus propiedades y dimensiones, con la finalidad de iniciar con la reagrupación de datos que se fracturaron durante la codificación abierta. Para Martínez (2009), las categorías se relacionan con sus subcategorías para formar explicaciones precisas y completas sobre los fenómenos. En esta fase, se analizaron los códigos resultantes de la codificación abierta y se agruparon por categorías, subcategorías, propiedades y dimensiones, lo cual resultó en una mejor comprensión del escenario bajo estudio. En la tabla 2 se muestran datos resultantes del proceso de codificación axial; encontrándose tres categorías principales, tres subcategorías, seis propiedades y veinte y nueve dimensiones.

Tabla 2: Fragmento de codificación axial del análisis de datos para describir los medios de comunicación para la apropiación del conocimiento

\begin{tabular}{|c|c|c|c|}
\hline Categorías & Subcategorías & Propiedades & Dimensiones \\
\hline \multirow{13}{*}{$\begin{array}{l}\text { Medios de } \\
\text { comunicación } \\
\text { educativa. }\end{array}$} & \multirow{13}{*}{$\begin{array}{l}\text { Medios } \\
\text { digitales. }\end{array}$} & \multirow{11}{*}{$\begin{array}{l}\text { Medios } \\
\text { audiovisuales. }\end{array}$} & Páginas web. \\
\hline & & & Aplicaciones para teléfonos inteligentes. \\
\hline & & & Tabletas. \\
\hline & & & Juegos en red. \\
\hline & & & CD-ROM. \\
\hline & & & DVD. \\
\hline & & & Televisión web. \\
\hline & & & Libros electrónicos. \\
\hline & & & Blogs. \\
\hline & & & Wikis. \\
\hline & & & Redes sociales. \\
\hline & & \multirow{2}{*}{$\begin{array}{l}\text { Medios } \\
\text { radiofónicos. }\end{array}$} & Radio digital. \\
\hline & & & Audios digitales. \\
\hline \multirow{8}{*}{$\begin{array}{l}\text { Proceso de } \\
\text { comunicación. }\end{array}$} & \multirow{8}{*}{$\begin{array}{l}\text { Interacción } \\
\text { sincrónica / } \\
\text { asincrónica. }\end{array}$} & \multirow{8}{*}{$\begin{array}{l}\text { Elementos de } \\
\text { interacción. }\end{array}$} & Emisor. \\
\hline & & & Receptor. \\
\hline & & & Mensaje. \\
\hline & & & Canal. \\
\hline & & & Código. \\
\hline & & & Contexto. \\
\hline & & & Barreras. \\
\hline & & & Retroalimentación (sincrónica / asincrónica). \\
\hline \multirow{8}{*}{$\begin{array}{l}\text { Alfabetización } \\
\text { tecnológica. }\end{array}$} & \multirow{8}{*}{$\begin{array}{l}\text { Desarrollo de } \\
\text { competencias } \\
\text { tecnológicas. }\end{array}$} & \multirow{4}{*}{$\begin{array}{l}\text { Competencias } \\
\text { instrumentales. }\end{array}$} & Habilidades cognitivas. \\
\hline & & & Capacidades metodológicas. \\
\hline & & & Destrezas tecnológicas. \\
\hline & & & Destrezas lingüísticas. \\
\hline & & \multirow{2}{*}{$\begin{array}{l}\text { Competencias } \\
\text { interpersonales. }\end{array}$} & Competencias para tareas en grupo. \\
\hline & & & Compromiso con el trabajo. \\
\hline & & \multirow{2}{*}{$\begin{array}{l}\text { Competencias } \\
\text { sistémicas. }\end{array}$} & Capacidades cognitivas. \\
\hline & & & Destrezas prácticas y disposiciones. \\
\hline
\end{tabular}

Las categorías se definen a continuación: (1) Medios de comunicación educativa: concentra los medios digitales en el mercado escolar: audiovisuales (páginas web, aplicaciones para teléfonos inteligentes, tabletas, juegos en red, CD-ROM, DVD, televisión web, libros electrónicos, blogs, wikis y redes sociales) y los radiofónicos (radio digital y audios digitales); (2) Proceso de comunicación: posibilitan la interacción sincrónica / asincrónica mediante sus elementos (emisor, receptor, mensaje, canal, código, contexto, barreras y retroalimentación(sincrónica/asincrónica), para la apropiación del conocimiento; y la (3) Alfabetización tecnológica: involucra el desarrollo de competencias tecnológicas que debe poseer el docente en esta nueva era global para la apropiación del conocimiento, como las competencias instrumentales (habilidades cognitivas, capacidades metodológicas, destrezas tecnológicas y lingüísticas), interpersonales (para tareas en grupo y compromiso con el trabajo), y sistémicas (capacidades cognitivas y destrezas prácticas y disposiciones). 


\section{RESULTADOS}

Terminado el análisis de la codificación abierta y axial, se comenzó el proceso de integrar y refinar la teoría (codificación selectiva). Strauss y Corbin (2008) y Martínez (2009), manifiestan que la integración de la teoría requiere la interacción constante analista-datos, iniciando desde la selección de una categoría medular que representa el tema principal de la investigación. En este caso las preguntas de investigación no cambiaron conforme avanzaba el análisis, los datos continuaron relacionados con las mismas preguntas. Se eligió la tercera categoría como núcleo central para darle respuesta a las preguntas de investigación planteadas, es decir, la categoría sobre la "medios de comunicación educativa". En el proceso del análisis de datos mediante las fases de codificación abierta, axial y selectiva, los códigos resultantes se clasificaron en tres categorías principales: (a) medios de comunicación educativa, (b) proceso de comunicación, y (c) alfabetización tecnológica, que interactuaban con algunas subcategorías: medios digitales, interacción sincrónica / asincrónica, y desarrollo de competencias tecnológicas. Al elegir la categoría central, se reordenaron las relaciones entre categorías, subcategorías, propiedades y dimensiones, quedando la categoría sobre los medios de comunicación educativa una relación con los procesos de comunicación, y la alfabetización tecnológica.

\section{Descripción de los medios de comunicación educativa}

Para esta investigación se contempló aspectos mencionados por los informantes claves, acciones o conductas multicaules, manifestadas en las vivencias tanto en el aula como en su entorno, detectándose la categoría central: "medios de comunicación educativa". Dicha categoría, en relación con las otras dos permiten responder las preguntas de investigación. La categoría central se vincula a los medios en espacios de aprendizajes para generar competencias (utilización, interacción y participación) del ser humano en sesiones de aprendizajes significativos. Se reportó la sub categoría: medios digitales, relacionadas con los medios audiovisuales y radiofónicos. En la figura 1 se observa que los docentes describen los medios de comunicación educativa como herramientas de aplicaciones móviles, comunicación productiva sincrónica y asincrónica, y redes socio técnicas.

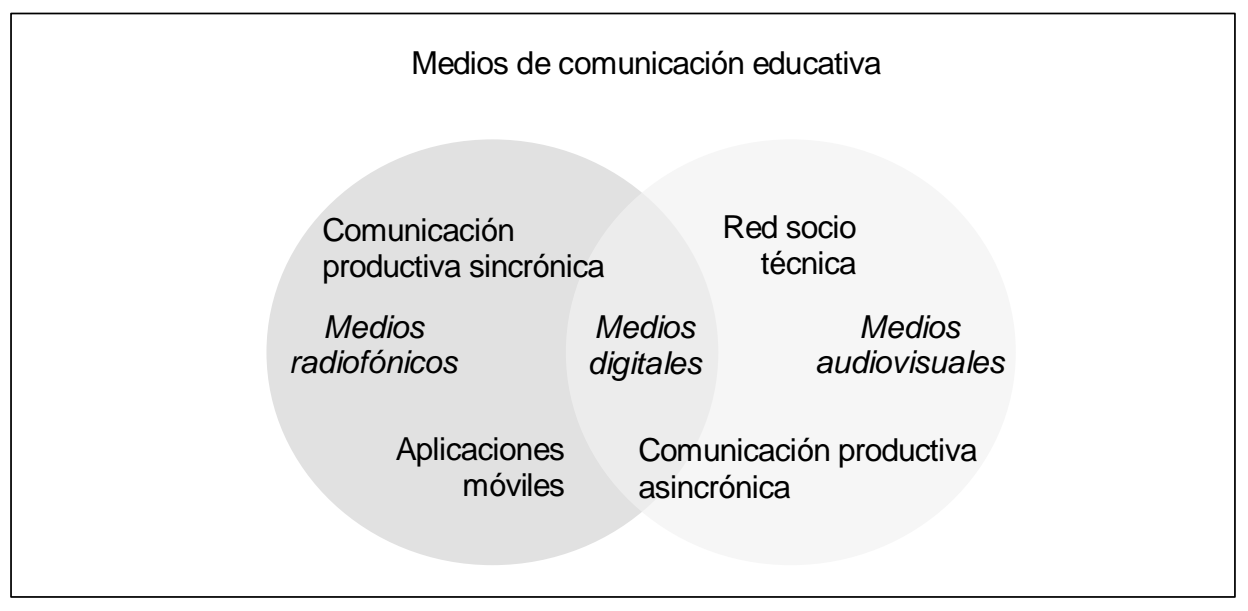

Fig. 1: Diagrama de Venn sobre la descripción de los medios de comunicación educativa

Los docentes entrevistados coincidieron en que los medios de comunicación educativa son necesarios para apropiar conocimiento, sin desmejorar los antiguos. Según, R7 hizo énfasis en la relación directa que existe al utilizar adecuadamente los gestos, articular y expresarse bien ante sus alumnos, calificando que el docente es el principal medio para apropiar conocimiento, y que ellos deben aprender estrategias para transmitir de una manera sencilla los conocimientos, en busca de aprendizajes significativos: "en oportunidades el docente se agota y recurre a otros medios tecnológicos para mantener la atención del estudiante, pero en mi caso al no contar con estos recursos me esfuerzo por implementar estrategias en los salones de clases para transmitir novedosamente mis conocimientos, y hacer que mis alumnos me entiendan, a veces en la escuela me facilitan una tableta, objetos virtuales de aprendizaje y los aprovecho cuando se puede, claro está, los estudiantes se emocionan más con estos recursos audiovisuales y captan más rápido lo que quiero transmitir, lo que hace que me desgaste menos" (R7). Por otro lado, R9 menciona que, para los docentes, los medios digitales "son importante por el valor que le puedes dar al proceso de enseñanza - aprendizaje, permitiendo la interacción sincrónica y asincrónica con el educando, además de adaptarse al nuevo uso de las herramientas digitales, como las páginas web, aplicaciones para teléfonos inteligentes, juegos en red, libros electrónicos y blogs" (R9).

En cuanto a la percepción de los docentes sobre los nuevos medios de comunicación social para la apropiación del conocimiento, R4 comenta: "considero muy importante la aparición de los nuevos medios de comunicación 
para utilizarse en las escuelas, ya que estos facilitan la transferencia de conocimiento, lo que podría aprovecharse para crear redes socio técnicas para la apropiación social del aprendizaje y conformación de comunidades de práctica: saber, saber hacer y saber ser, transformando integralmente al individuo" (R4). En el mismo sentido, R1 afirma que el usar medios digitales en las sesiones de aprendizajes "sí, impacta positivamente el proceso para apropiar conocimiento, pero es necesario que a las instituciones de educación se les dote de estas herramientas tecnológicas, y a su vez se forme al personal docente para su adecuado uso...parece sorprendente, pero en oportunidades el docente no sabe cómo usar las tecnologías de la comunicación en el aula de clases" (R1). Sin embargo, las opiniones de los docentes coinciden en que la evolución de los medios de comunicación social para la apropiación del conocimiento emerge tan rápido, que esto originan brechas de conocimiento en cuanto al uso se refiere, tanto del docente como de los estudiantes. R1 asegura que "al aparecer nuevos medios de comunicación social para apropiar conocimiento, se origina nuevas formas de gestionar el aprendizaje, impactando socialmente el currículo y modelo escolar, donde el aprendizaje es inmediato, compartido, holístico, sistémico e innovador" (R1). Es decir, ya el apropiar conocimiento solamente no depende del docente, ya que se habla de coaprendizaje, mediante la autoformación, formación, producción integrada y colaborativa, de saberes y conocimiento. Por su parte, R7 comenta porqué piensa que los medios educativos para apropiar conocimiento estimulan el aprendizaje de los estudiantes: "mis estudiantes se muestran más interesados, atentos y participan más cuando incorporo algún medio audiovisual o radiofónico, eso los motiva a interactuar" (R7).

Por otro lado, los docentes hicieron énfasis en que los medios de comunicación educativa, permiten la creación de redes de innovación y aprendizaje. R4 justifica que la innovación para la apropiación del conocimiento genera nuevas maneras de aprender "la nueva era de la innovación y conocimiento está haciendo que cambiemos nuestras maneras de enseñar y aprender, que creemos redes de saberes, entre las demás escuelas para que tanto nosotros los docentes como los estudiantes podamos desaprender para aprender y así emprender" (R4). Las opiniones entre los docentes son muy similares, de acuerdo a su experiencias y percepciones con los que cuentan. En ese sentido, la categoría sobre descripción de los medios de comunicación educativa, se ve relacionada con los medios audiovisuales y radiofónicos. Los docentes consideran importante la oportunidad de generar nuevos conocimientos con estos recursos tecnológicos, el siguiente fragmento de la entrevista a R1 describe los medios educativos: "los nuevos tiempos que vivimos nos han permitido que incluyamos dentro de nuestras sesiones de aprendizajes la radio digital, audios digitales, páginas web, aplicaciones para teléfonos inteligentes, tabletas, juegos en red, CD-ROM, DVD, televisión web, libros electrónicos, blogs, wikis y redes sociales...todo esto captura más la atención de los estudiantes, ya que ellos están envueltos en entornos de su vida que limitan el desarrollo de sus competencias, vinculados a factores económicos y sociales que complejizan la dinámica de clases para que estos apropien su cocimiento" (R1).

\section{Proceso de comunicación}

Los docentes vinculados a las instituciones educativas de los Municipios sin certificarse del Departamento del Magdalena, que fueron entrevistados consideran como medios de comunicación educativa los: radiofónicos y audiovisuales, originados por la era digital. En la sección anterior se expusieron los motivos por los cuales dichos medios son catalogados como referentes al hablar de medios educativos, procurando la comunicación productiva tanto asincrónica como sincrónica, lo que posibilita a las comunidades educativas el uso de las aplicaciones móviles y establecimiento de redes socio técnicas. R7 comenta que el proceso de comunicación que se establece en la institución educativa depende de cada docente, es decir, no existe un modelo de comunicación educativa donde el docente se oriente para que los estudiantes apropien el conocimiento: "Pues yo digo que cada docente crea su propio proceso de comunicación para dar clases, esto depende de cada docente, y varía de acuerdo a su edad, contenido de clases, número de participantes, herramientas didácticas disponibles, estructura de la institución, condiciones eléctricas, y factores ambientales, como el clima, aunque..." (R7).

Al respecto el tema de las instalaciones de las instituciones educativas es considerada como crucial, ya que afectarían positiva o negativamente el proceso de comunicación para la apropiación de conocimiento, originando barreras y filtros que limitan el aprendizaje significativo: “. . en oportunidades debido a lluvias, falta de electricidad e inadecuadas instalaciones de la institución, perjudican nuestra labor en el aula, limitando el proceso de comunicación para la enseñanza-aprendizaje... también me he podido percatar que se carece de estrategias pedagógicas para establecer interacciones activas y participativas entre los estudiantes, lo que hace que el discurso del docente sea menos atractivo y poco innovador, lo que desmotiva la retroalimentación en las sesiones de aprendizaje" (R1). El proceso de comunicación para la apropiación del conocimiento en instituciones educativas del departamento de Magdalena, depende de la experiencia previa con la que cuente el docente en cuestión. Es decir, cuando el docente posee más experiencia en su labor, procura prever con que herramientas contar para que en su proceso de comunicación, al enseñar sea más significativo el aprendizaje. R1 manifiesta que su experiencia con el proceso de enseñanza aprendizaje con sus estudiantes ha sido novedosa y satisfactoria: "tengo 12 años trabajando como docente, y mi experiencia ha sido buena puesto que por cada mes planifico adecuadamente mis sesiones de aprendizajes y establezco los recursos a utilizar, y si no tengo las 
herramientas tecnológicas las busco con tiempo para evitar contratiempos... me he percatado que cuando realizo actividades donde quiero que mis alumnos participen cada uno, debo apoyarme con videos, audios, tabletas, y de esta manera la comunicación es sincrónica... de igual manera es necesario limitar las funciones del emisor, receptor, mensaje, canal, código, contexto y barreras en las clases, porque así cada uno de los actores dentro del procesos de aprendizaje se adapta a su rol, lo que origina que se dé una adecuada apropiación del conocimiento" (R1).

También mencionan cómo el proceso de comunicación que utilizan los docentes, ha influido en la apropiación de conocimiento de los estudiantes, haciendo énfasis en la creación de redes de innovación y aprendizaje: "el establecer procesos de comunicación educativo permite innovar y generar nuevos conocimientos, lo que se puede replicar a otras instituciones educativas, a fin de garantizar estos resultados ... Es decir, se podrían implementar las mejores prácticas docentes, con la intervención de herramientas tecnológicas en otras escuelas, y a su vez detectar cualquier falencia dentro del procesos de enseñanza-aprendizaje, para garantizar así la apropiación del conocimiento por parte de los estudiantes" (R1). Si el docente establece un adecuado proceso de comunicación, se podría garantizar la calidad del aprendizaje. R7 expone que su experiencia con los estudiantes ha sido satisfactoria: "Mi experiencia ha sido buena, pues se observa en los estudiantes un alto rendimiento en sus competencias, y además un desenvolvimiento idóneo en su proceso de aprendizaje, adaptado a las necesidades del contexto, lo que ha impactado positivamente la calidad de vida de estos..." (R7). Debido a ello, se puede percibir una notoria relación entre los medios de comunicación educativa y la utilización del proceso de comunicación para la apropiación del conocimiento que ejerce el docente: "es evidente que el sistema educativo debe adaptarse a la era actual, que los medios de comunicación se han convertido en una herramienta para gestionar aprendizajes, y que el docente debe diseñar procesos mediadores para garantizar la apropiación de estos... al mismo tiempo contar con competencias tecnológicas que permitan la creación de comunidades escolares, participativa y autodidactas...estas estrategias permiten el avance de los alumnos, así como el progreso de un país que se ha quedado estancado en cuestión a la práctica docente, porque muchos de los maestros se han quedado sin preparación alguna" (R7).

\section{Alfabetización tecnológica}

Los docentes que fueron entrevistados no mencionan la resistencia ni la falta de competencias de los estudiantes para el uso de los medios de comunicación educativa, más bien se basan en la accesibilidad de dichos recursos, falta de cultura tecnológica docente-estudiante y adecuadas instalaciones (eléctricas) para establecer procesos de comunicación con medios educativos para apropiar conocimiento, en la sección anterior se expusieron los motivos por los cuales estas situaciones prevalecen y son constantes en las escuelas. R9 comenta que no basta con contar con recursos tecnológicos, también influye la falta de competencias tecnológicas tanto del docente como de los alumnos, porque en ocasiones se hace necesario apoyarse con las tecnologías de la información y comunicación para consolidar los procesos de aprendizajes, a fin de que estos se apropien: "yo creo que sería más importante antes de dotar a las escuelas de medios tecnológicos, adiestrar al docente y alumnos, para el adecuado uso de los mismos... por tal motivo se hace necesario vincular primeramente al docente y hacer énfasis en cómo aplicar los medios de comunicación educativa en las estrategias de aprendizajes, y así integrarlos en las sesiones de saberes para apropiar conocimiento" (R9).

La alfabetización tecnológica, busca entender y utilizar la información para apoyar el aprendizaje, productividad personal, toma de decisiones y la vida diaria, y esto varía de acuerdo a la experiencia de cada docente. Es decir, dependiendo del tiempo que posee el docente en su ejercicio, esto redundará en el grado de experticia que este tenga, constante similar a los resultados con la categoría proceso de comunicación, lo que fortalecería la apropiación de conocimiento en los estudiantes. Al respecto esto procura el desarrollo de competencias al modelo de aprendizaje adaptado a la era digital, entre estas las: instrumentales, interpersonales y sistémicas. El participante R9 quién posee una mayor experiencia de 16 años en cuanto al ejercicio docente menciona lo siguiente: "es muy importante...que el docente se adapte día tras día a la era digital, al uso de los medios de comunicación educativa para la apropiación de concomiendo, siendo el docente el principal medio para consolidar aprendizaje. También, no se puede olvidar que al adquirir competencias, se tendrá la capacidad de crear e innovar nuevas maneras de aprender, mediante el desarrollo de prácticas pedagógicas, involucrando a todos los actores: escuela, comunidad escolar y familia... en oportunidades veo más resistencia entre los docentes al uso de estos medios de comunicación..." (R9).

Al respecto la alfabetización tecnológica, en los docentes de las instituciones educativas del departamento de Magdalena - Colombia, referencia las habilidades, conocimientos y capacidades, para el uso adecuado de productos o sistemas tecnológicos, mejorando el desempeño del individuo. R4 posee conocimientos básicos sobre el tema y comenta lo siguiente al respecto: “....sería secundario hablar de las adecuadas instalaciones que carecen las escuelas donde trabajamos, para mí lo necesario es adaptarnos primero al uso de los medios de comunicación educativo, que por factores sociales, políticos, económicos y sociales, pocos de los docentes 
hemos tenido ese alcance, y por eso se han experimentado pocas sesiones de aprendizaje vinculando los recursos tecnológicos para tal fin, así sepamos sus fortalezas..." (R4).

\section{DISCUSIÓN}

En el proceso de revisión literaria de la investigación, se hallaron investigaciones en donde los autores emplean este método de análisis de datos cualitativos, como es el caso de la teoría fundamentada, para que las personas puedan describir un fenómeno de estudio de acuerdo a temas de interés en diversas áreas como el de las ciencias sociales (Sandín, 2003; Martínez, 2009), psicología (Strauss y Corbin, 2008), gestión empresarial (Martínez-Salgado, 2012), tecnología, estudio de mercados (Glaser y Strauss, 2017), por nombrar algunos. Al mismo tiempo, Sandín (2003) y Martínez (2009), propone una serie de estrategias para llevar a cabo un análisis cualitativo en el contexto educativo, lo que facilitó la descripción de los medios de comunicación para la apropiación del conocimiento en instituciones educativas del departamento de Magdalena - Colombia.

El análisis obtenido de este estudio indica que todos los individuos entrevistados coincidieron en que los medios de comunicación educativa son necesarios actualmente para apropiar conocimiento en los estudiantes, vinculado a los medios audiovisuales y radiofónicos (radio digital, audios digitales, páginas web, aplicaciones para teléfonos inteligentes, tabletas, juegos en red, CD-ROM, DVD, televisión web, libros electrónicos, blogs, wikis y redes sociales). Los docentes consideran importante la oportunidad de generar nuevos conocimientos con estos recursos tecnológicos, sin quitarle mérito a los recursos convencionales, enfatizando que el docente debe utilizar adecuadamente gestos y expresarse bien ante sus alumnos, siendo este el principal medio para apropiar conocimiento. Los medios digitales, otorgan plusvalía al proceso de enseñanza - aprendizaje, posibilitando la interacción sincrónica y asincrónica con el estudiante, transferencia de conocimiento, lo que podría aprovecharse para crear redes socio técnicas y conformación de comunidades de práctica: saber, saber hacer y saber ser, transformando integralmente al individuo.

Es necesaria la dotación de herramientas tecnológicas en las escuelas y a su vez se forme al personal docente para su adecuado uso, ya que al evolucionar los medios de comunicación se han originado brechas de conocimiento, tanto del docente como de los estudiantes, lo que impacta socialmente el currículo y modelo escolar, donde el aprendizaje es inmediato, compartido, holístico, sistémico e innovador. Los medios de comunicación educativa, son novedosos canales utilizados por educadores y estudiantes para propiciar conocimiento, presencial, corporal o verbal, virtuales (Cabero y Llorente, 2010; Gómez-Rodríguez et al., 2017). Generalmente implica el uso de las TIC, como el convencional recurso o herramienta educativa, sincrónico y asincrónico. Sugiere el paso a dispositivos móviles, ordenadores de última generación con acceso a internet, televisores, video proyectores, infraestructura de telecomunicaciones al servicio de la educación (Mullan, 2018; Määttä et al., 2017).

El proceso de comunicación que se establece en la institución educativa depende de cada docente, no existe un modelo de comunicación educativa para que los estudiantes apropien el conocimiento, esto depende de cada docente, y varía de acuerdo a su edad, contenido de clases, número de participantes, herramientas didácticas disponibles, estructura de la institución, condiciones eléctricas, y factores ambientales, como el clima, originando barreras y filtros que limitan el aprendizaje significativo, todo esto aunado a la carencia de estrategias pedagógicas para establecer interacciones activas y participativas entre los estudiantes, lo que hace que el discurso del docente sea menos atractivo y poco innovador, desmejorando la retroalimentación en las sesiones de aprendizaje. De igual manera es necesario articular sistémicamente las funciones del emisor, receptor, mensaje, canal, código y contexto en las clases, porque así cada uno de los actores dentro del procesos de aprendizaje se adapta a su rol, mitigando las barreras ya expuestas, procurando redes de innovación y generar nuevos conocimientos, redundando en las mejores prácticas docentes. Para Klapper (1957), el proceso de comunicación contribuye a la participación en redes de conocimiento y aprendizaje innovador, aumentando la capacidad de relacionamiento e información, entre las comunidades de saber, conocimiento y transformación. Esto enriquece las actividades de trabajo colaborativo entre los estudiantes y conseguir el desarrollo de competencias transversales en la práctica educativa, como el trabajo en equipo, autonomía, respeto a lo multiétnico, derecho de autor, responsabilidad del discurso (Rodríguez-Hoyos y Fueyo, 2018; Castro-García et al., 2016).

La alfabetización tecnológica, busca entender y utilizar la información para apoyar el aprendizaje, productividad personal, toma de decisiones y la vida diaria, dependiendo del tiempo que posee el docente en su ejercicio, esto redundará en el grado de experticia que este tenga, constante similar a los resultados con la categoría proceso de comunicación, lo que fortalecería la apropiación de conocimiento en los estudiantes, esto procura el desarrollo de competencias al modelo de aprendizaje adaptado al tecnoconocimiento, entre estas las: instrumentales, interpersonales y sistémicas. Reflejando: (a) apoyo al aprendizaje estudiantil creativo, (b) experiencias de aprendizaje propias a los medios educativos y (c) competencias al modelo de aprendizaje adaptado a la era digital. Vinculada a todo lo que el ser humano elabora y concibe (aprendizaje, 
experiencias, uso), para satisfacer requerimientos, proporcionando servicios idóneos en entornos artificiales, físicos o virtuales. Por tal motivo, permite desarrollar conocimientos y habilidades tanto instrumentales como cognitivas en relación con la información vinculada a través de nuevas tecnologías (manejar el software, buscar información, enviar y recibir correos electrónicos, utilizar los distintos servicios web), además plantear y desarrollar valores y actitudes de naturaleza social-ambiental con relación a las tecnologías.

El perfil del profesorado que desarrolla un modelo didáctico intensivo de las TIC es un docente con años de experiencia profesional, por tanto, se debe fortalecer el apoyo al aprendizaje estudiantil y creativo, experiencias de aprendizaje propias a los medios emergentes, competencias al modelo educativo y responsabilidad adaptado a la era digital (Pérez-Escoda et al., 2016; Fernández-Cruz y Fernández-Díaz, 2016; McLuhan, 2015). La tecnología en los espacios de aprendizajes, se debe orientar en la alfabetización y multialfabetismo técnico, son oportunidades de desaprendizaje, reaprendizaje, del proceso educativo permanente, favoreciendo la globalización cultural mediática, con miras a la productividad y ética en las TIC; aunque el acceso no es igual para todas las personas y que, aun accediendo, no todos participan plenamente (Eze et al., 2018; Pabón, 2017; Schwartz, 2015).

\section{CONCLUSIONES}

De acuerdo con los resultados obtenidos, se concluye que: (1) los medios de comunicación educativos se ven impactados por la cultura tecnológica adquirida del docente-estudiante, originando brechas de conocimiento para su adecuado uso y aprovechamiento para gestionar aprendizaje; (2) se encontró que los medios y proceso de comunicación se ven influenciados con la edad del docente-estudiante, contenido de clases, número de participantes, herramientas didácticas disponibles, condiciones de la estructura de la institución y factores ambientales, como el clima; originando barreras, desmotivación y filtros que limitan el aprendizaje significativo; (3) en la apropiación de conocimiento, de las instituciones educativas predominan los modelos interactivos, perspectivas emergentes sobre la metodología para el saber y su producción, afectada por la tecnología, y ésta varía por la cultura de su público, favorecedora del cambio socio-cognitivo, interacción a/simétrica, adaptándose a nuevas audiencias y desarrollo de modelos educativos en función a las nuevas tecnologías, competencias ciudadanas y éticas; (5) los medios de comunicación se hacen necesario actualmente, la era global, competitividad, innovación y conectividad, han originado que se consideren como prioritarios para la apropiación del conocimiento en las instituciones educativas, siendo ahora el reto, adaptarse a los nuevos cambios que todo esto acarrea.

\section{REFERENCIAS}

Area-Moreira, M., Hernández-Rivero, V., y Sosa-Alonso, J., Modelos de integración didáctica de las TIC en el aula, https://doi.org/10.3916/C47-2016-08, Comunicar, 47(XXIV), 79-87 (2016).

Bartau-Rojas, I., Aierbe-Barandiaran, A., y Oregui-González, E., Parental mediation of the Internet use of primary students: beliefs, strategies and difficulties, https://doi.org/10.3916/c54-2018-07, Comunicar, 54(1), 71-79 (2018).

Cabero Almenara, C., y Llorente, M. del C., Students' learning experience on the virtual andalusian campus, International Journal of Educational Technology in Higher Education, 7(1), 93-106 (2010).

Castro-García, D., Olarte, D., y Corredor,J., Technology for communication and problem solving in the classroom. Effects on significant learning, Digital Education Review, 30(1), 207-219 (2016).

Chassiakos, Y.R., Radesky, J., y otros 4 autores, Children and adolescents and digital media, https://doi.org/10.1542/peds.2016-2593, Pediatrics, 138(5), 1-19 (2016).

Eze, S., Chinedu-Eze, V., y Bello,A., Actors and emerging information, communications and technology (EICT) adoption: a study of uk small and medium services enterprises, https://doi.org/10.1080/23311975.2018.1480188, Cogent Business \& Management, 5(1), 1-19 (2018).

Félix, M.A., La comunicación en las teorías de las organizaciones. El cruzar del siglo XX y la revolución de las nuevas tecnologías. Una visión histórica, https://doi.org/10.5209/rev_HICS.2014.v19.45021, Revista Historia y Comunicación Social, 19 ( ${ }^{\circ}$ Esp.2014), 195-210 (2014).

Fernández-Cruz, F., y Fernández-Díaz, M., Los docentes de la Generación $Z$ y sus competencias digitales, https://doi.org/10.3916/C46-2016-10, Comunicar, 46(XXIV), 97-105 (2016).

Glaser, B., y Strauss, A., Discovery of grounded theory: strategies for qualitative research, $1^{a}$ edición, 001-282. Grupo Taylor \& Francis, Abingdon, Reino Unido (2017).

Gómez-Rodríguez, G., Morell, A. E., y Gallo-Estrada, C., A 30 años de comunicación y sociedad: cambios y permanencias en el campo académico de la comunicación, https://doi.org/10.32870/cys.v0i30.6838, Comunicación y Sociedad, 30(1), 17-44 (2017).

Jiménez-Iglesias, E., Garmendia-Larrañaga, M., y Río, M.C., Children's perception of the parental mediation of the risks of the Internet, https://doi.org/10.4185/RLCS-2015-1034, Revista Latina de Comunicación Social, 70 (1), 49-68 (2015). 
Klapper, J. T., What we know about the effects of mass communication: the brink of hope, Public Opinion Quarterly, 22 (4), 453-474 (1957).

Lee, A.S., A Commentary: theory appropriation and the growth of knowledge, https://doi.org/10.1016/j.jsis.2016.02.008, Journal of Strategic Information Systems, 25(1), 68-71 (2016).

Lin, L., y Wei, P., Transitioning through social media: International students' sns use, perceived social support, and acculturative stress, https://doi.org/10.1016/j.chb.2019.03.011, Computers in Human Behavior, 98(1), 69-79 (2019).

Livingstone, S., Mascheroni, G., y Staksrud, E., European research on children's internet use: assessing the past and anticipating the future, https://doi.org/10.1177/1461444816685930, New Media \& Society, 20(3), 1103-1122 (2018).

Määttä, S., Kaukonen, R., y otros 4 autores, The mediating role of the home environment in relation to parental educational level and preschool children's screen time: a cross-sectional study, https://doi.org/10.1186/s12889-017-4694-9, BMC Public Health, 17(1), 1-11 (2017).

Martín Serrano, M., La comunicación y la información en un mundo que se virtualiza. Desarrollos y funciones previsibles, https://doi.org/10.32870/cys.v2019i0.7478, Comunicación y Sociedad, e7478, 1-29 (2019).

Martínez-Salgado, C., El muestreo en investigación cualitativa: principios básicos y algunas controversias, https://doi.org/10.1590/S1413-81232012000300006, Ciência \& Saúde Coletiva, 17, 613-619 (2012).

Martínez, M.M., Epistemología y metodología cualitativa en las ciencias sociales, $2^{a}$ edición, 001-336. Editorial Trillas, S.A. de C.V, México, D.F (2009).

McQuail, D., Accountability of media to society. Principles and means, https://doi.org/10.1177/0267323197012004004, European journal of communication, 12(20), 511-529 (1997).

Mullan, K., Technology and children's screen-based activities in the uk: the story of the millennium so far, https://doi.org/10.1007/s12187-017-9509-0, Child Indicators Research, 11(6), 1781-1800 (2018).

Pabón, R., Apropiación social del conocimiento: una aproximación teórica y perspectivas para Colombia, https://doi.org/10.17081/eduhum.20.34.2629, Educación y Humanismo, 20(34), 116-139 (2017).

Pérez-Escoda, A., Castro-Zubizarreta, A., y Fandos-Igado, M., La competencia digital de la generación z: claves para su introducción curricular en la educación primaria, https://doi.org/10.3916/C49-2016-07, Comunicar, 49(XXIV), 71-80 (2016).

Prendes, E. y Gutiérrez, P., Technological skills of teachers in spanish universities, https://doi.org/10.4438/1988-592X-RE2011-361-140, Revista de Educación, 361(1), 196-222 (2013).

Rodríguez-Hoyos, C., y Fueyo, A., Media training for future education professionals: a study of the spanish context, Digital Education Review, 33(1), 217-234 (2018).

Sandín, E.M., Investigación cualitativa en educación: fundamentos y tradiciones, $1^{\text {a }}$ edición, 001-258. McGraw-Hill/ Interamericana de España, S.A.U, Madrid, España (2003).

Schwartz, L., A funds of knowledge approach to the appropriation of new media in a high school writing classroom, https://doi.org/10.1080/10494820.2015.1064448, Interactive Learning Environments, 23(5), 595-612 (2015).

Strauss, A., y Corbin, J., Pesquisa cualitativa: técnicas e procedimientos para o desenvolvimiento de teoría fundamentada, $1^{\text {a }}$ edición, 001-181. Artmed, Porto Alegre, Brasil (2008).

Valcke, M., Bonte, S., Wever, B.D., y Rots,I., Internet parenting styles and the impact on Internet use of primary school children, https://doi.org/10.1016/j.compedu.2010.02.009, Computers \& Education, 55(2), 454-464 (2010).

Villalobos Antúnez, J.V., Ramírez Molina, R.I. y Díaz-CID, L., Bioética y biopoder: perspectivas para una praxis pedagógica desde la ética de álvaro márquez-fernández, https://doi.org/10.5281/zenodo.346378, Utopía y Praxis Latinoamericana, Revista Internacional de Filosofía y Teoría Social, 24(87), 65-77 (2019). 
